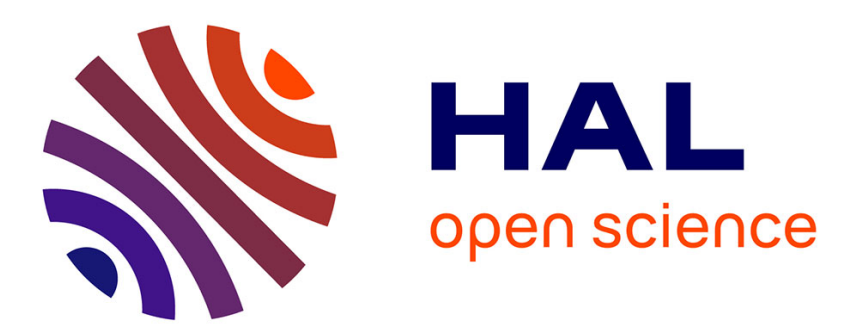

\title{
Forecasting the 2016 US Presidential Elections Using Sentiment Analysis
}

Prabhsimran Singh, Ravinder Singh Sawhney, Karanjeet Singh Kahlon

\section{To cite this version:}

Prabhsimran Singh, Ravinder Singh Sawhney, Karanjeet Singh Kahlon. Forecasting the 2016 US Presidential Elections Using Sentiment Analysis. 16th Conference on e-Business, e-Services and eSociety (I3E), Nov 2017, Delhi, India. pp.412-423, 10.1007/978-3-319-68557-1_36 . hal-01768531

\section{HAL Id: hal-01768531 \\ https://hal.inria.fr/hal-01768531}

Submitted on 17 Apr 2018

HAL is a multi-disciplinary open access archive for the deposit and dissemination of scientific research documents, whether they are published or not. The documents may come from teaching and research institutions in France or abroad, or from public or private research centers.
L'archive ouverte pluridisciplinaire HAL, est destinée au dépôt et à la diffusion de documents scientifiques de niveau recherche, publiés ou non, émanant des établissements d'enseignement et de recherche français ou étrangers, des laboratoires publics ou privés.

\section{(c)(1)}

Distributed under a Creative Commons Attribution| 4.0 International License 


\title{
Forecasting the 2016 US Presidential Elections using Sentiment Analysis
}

\author{
Prabhsimran Singh ${ }^{1}$, Ravinder Singh Sawhney ${ }^{2}$, Karanjeet Singh Kahlon ${ }^{3}$ \\ ${ }^{1,3}$ Department of Computer Science, ${ }^{2}$ Department of Electronics Technology, \\ 1,2,3 Guru Nanak Dev University, Amritsar, India. \\ 1prabh_singh32@yahoo.com, ${ }^{2}$ sawhney.ece@gndu.ac.in, ${ }^{3}$ karanvkahlon@yahoo.com
}

\begin{abstract}
The aim of this paper is to make a zealous effort towards true prediction of the 2016 US Presidential Elections. We propose a novel technique to predict the outcome of US presidential elections using sentiment analysis. For this data was collected from a famous social networking website (SNW) Twitter in form of tweets within a period starting from September 1, 2016 to October 31, 2016. To accomplish this mammoth task of prediction, we build a model in WEKA 3.8 using support vector machine which is a supervised machine learning algorithm. Our results showed that Donald Trump was likely to emerge winner of 2016 US Presidential Elections.
\end{abstract}

Keywords: Forecasting, Twitter, Sentiment Analysis, Support Vector Machine, WEKA.

\section{Introduction}

Accurate future prediction of an event has always been a tedious task for researchers, but with advancement in technologies and availability of powerful computing devices researchers have started taking keen interest in this research area. One of the key factor in these advancement has been the popularity of social networking websites (SNW) especially Twitter. Twitter is one of the most popular social networking media, with 695,750,000 registered users till date and approximately 135,000 new users are registering every day [1]. This large audience is responsible for tons of tweeting happening everyday i.e. sharing their view in relatively fewer words and hence providing researchers a large pool of tweets, which may contain anger or love towards an entity like an election. Using the concept of sentiment analysis as suggested by Liu [2], we can extract their sentiments from these tweets and use these in predicting the outcome of any event, be it elections. Since US is a developed country [3], with an established fact that $88.5 \%$ of the population has access to the internet [4] and approx 67 million Twitter users in the US [5], all these factors give us a perfect platform to carry out our research on 2016 US Presidential Elections.

For this research paper, we have collected the tweets through Twitter. Then we synthesized these tweets using sentiment analysis that helped us to have a better insight into the outcome of 2016 US Presidential Elections. We would be discussing our approach towards our predicted results in the upcoming sections. 


\section{Background of US Presidential Elections}

US Presidential elections were scheduled to be held on November 8, 2016 to elect the new President of United States of America for the next 4 years, as the second term of the current President Mr. Barack Obama was going to expire on January 2017. Since Obama was holding the presidential chair for the second term, so as per the US presidential ordinances he could not contest these elections. The event became more engaging, as both the candidates contesting the election were first timers. As we know Democratic Party and Republican Party were the two main parties, so the entire paper has been focused on these parties as well as their Presidential candidates.

The selection of both presidential candidates was made through primaries held between February to June 2016. In the Democratic Party Presidential primaries Ms. Hillary Clinton defeated Mr. Bernie Sanders, thus becoming the first female Presidential candidate in the history of United States, to be nominated by a major political party. While the Republican Party Presidential primaries saw 17 candidates were entering the primaries, making it the largest ever presidential primary contesting for any political party in United States history. In the finals Mr. Donald Trump, a businessman manages to defeat Mr. Ted Cruz to be selected as Republican Party Presidential candidate.

None of the candidates had an absolute cakewalk, and both faced their respective ups and downs during the course of their campaign and debates. Donald Trump had easy primaries while Hillary Clinton had a tough fight with Bernie Sanders. During debates, Hillary Clinton always had an edge over Donald Trump. Donald Trump was highly criticized for various comments and attitude toward other nations during campaigns and speeches while Hillary Clinton had tough times for her email controversies. So even up to week before the elections, there was ambiguity about the winner and the lead was constantly swinging among both candidates.

\section{Related Work}

Twitter and Elections share a strong bond since a longtime now. With advancement in technology and increase in a number of people using Twitter, the researchers working in this domain have a perfect opportunity to work on Twitter based emotions towards election predictions. Though this approach was rather crude and had many flaws yet it provided useful insights that helped us towards making a realistic prediction with some modern prediction tools the task seems realistic.

Tumasjan et al. [6] were the first to make use of Twitter to predict the results of German Federal election held in September 2009. They collected 104,003 tweets over the period of 27 days for the six popular political parties of Germany. Their technique was quite simple and dependent on a basic counting of the number of tweets that a party or its prominent leaders get. Using this simple technique, they were successful in predicting the winner of 2009 German Federal Elections. However this simple 
technique faced huge criticism, in particular, Jungherr et al. [7] pointed the lack of methodological justification while Gayo-Avello [8-9] stressed on the need to make true prediction i.e. predictions made prior to the actual election. Another point highlighted by Gayo-Avello [8-9] was to make use of sentiment analysis in order to know the sentiment of the tweet, which indeed will help to produce more accurate results. The subsequent studies DiGrazia et al. [10], Franch [11], Ceron et al. [12], Caldarelli at al. [13], Burnap et al. [14] have all taken the advice of Gayo-Avello and made use of sentiment analysis in order to produce more accurate results.

Our work is also influenced by the advice of Gayo-Avello [8-9]. We made a true prediction for 2016 US Presidential elections, instead of simply relying on the amount of tweets for making the prediction we have used sentiment analysis in our methodology along with some scientific tools to make predictions.

\section{Proposed Methodology}

Data collection is a trivial task and in our case as well the initial hurdle was efficient data collection. So we gathered data from Twitter in form of tweets. For this, we built a system in ASP.Net 2012. Since a person can post multiple tweets on Twitter, so in order to avoid biased results, we have first removed multiple tweets from single source so that only one tweet could be considered from one person. Next we applied sentiment analysis to obtain polarity (positive or negative) of each tweet using WEKA 3.8. All these phases are discussed with suitable explanation in the upcoming sections. The flowchart of the process is given in Figure 1.

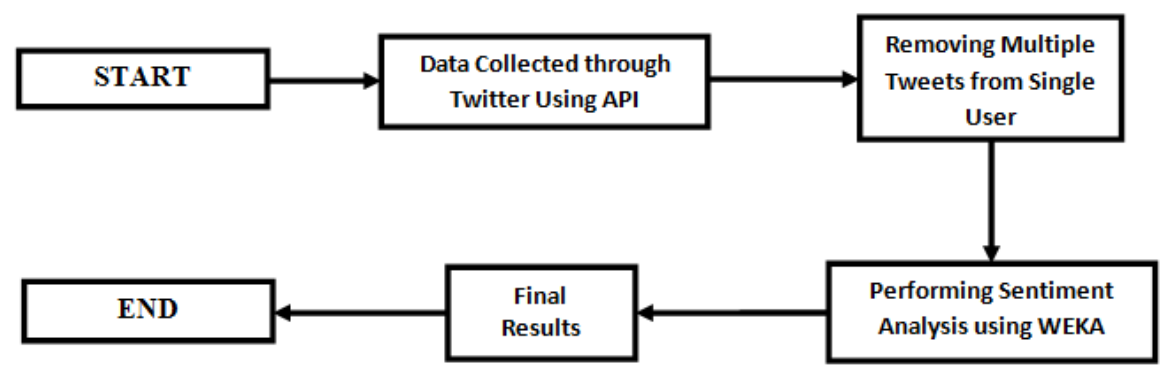

Fig. 1. Flowchart of proposed Methodology

\section{Data Collection}

Data for our research was collected from Twitter. For this purpose, a system was developed in ASP.Net using visual studio [15]. For tweet fetching we used tweetinvi API [16] which is freeware and can be easily integrated with Dot.Net framework. The tweets were fetched using this system based on the hashtags (\#) for both the 
respective candidates. Table 1 shows the hashtags (\#) that were used for fetching tweets from Twitter.

Table 1. Hashtags (\#) used for fetching tweets

\begin{tabular}{|c|c|c|}
\hline Candidates & Hillary Clinton & Donald Trump \\
\hline \multirow{2}{*}{ Hashtags(\#) } & $\begin{array}{c}\text { \#Hillary, \#HillaryClinton, } \\
\text { \#ClintonKaine, \#Votehillary }\end{array}$ & $\begin{array}{c}\text { \#DonaldTrump, \#TrumpPence16, } \\
\text { \#Trump, \#VoteTrump }\end{array}$ \\
\hline
\end{tabular}

A total of 327,127 tweets were collected from September 1, 2016 to October 31, 2016 daily from the USA. This time period was chosen because the election campaigns were in full swing so it was possible to get data from all type of Twitter users at this time. Out of the 327,127 tweets collected from the USA, 194,753 (59.53\%) of tweet mentions were in favor of Donald Trump, while 132,374 (40.47\%) of tweet mentions were in favor of Hillary Clinton. Table 2 shows the daily tweet collection for both the candidates.

Table 2. Daily tweet collection for both candidates

\begin{tabular}{|c|c|c|}
\hline Date & Donald Trump & Hillary Clinton \\
\hline $01-09-16$ & 3512 & 1356 \\
\hline $02-09-16$ & 2728 & 1499 \\
\hline 03-09-16 & 2757 & 1329 \\
\hline 04-09-16 & 2319 & 1160 \\
\hline $05-09-16$ & 2548 & 1122 \\
\hline $06-09-16$ & 2361 & 2638 \\
\hline 07-09-16 & 2722 & 1520 \\
\hline $08-09-16$ & 3587 & 2193 \\
\hline 09-09-16 & 2638 & 1475 \\
\hline $10-09-16$ & 1919 & 1644 \\
\hline $11-09-16$ & 2410 & 4649 \\
\hline $12-09-16$ & 2748 & 1667 \\
\hline $13-09-16$ & 2152 & 1552 \\
\hline $14-09-16$ & 2661 & 1596 \\
\hline $15-09-16$ & 3483 & 1803 \\
\hline $16-09-16$ & 3771 & 2027 \\
\hline $17-09-16$ & 3369 & 1554 \\
\hline $18-09-16$ & 3184 & 1548 \\
\hline $19-09-16$ & 2280 & 1604 \\
\hline $20-09-16$ & 2818 & 1678 \\
\hline 21-09-16 & 3253 & 4226 \\
\hline $22-09-16$ & 2960 & 1524 \\
\hline $23-09-16$ & 2902 & 1392 \\
\hline 24-09-16 & 2617 & 1454 \\
\hline $25-09-16$ & 2729 & 1640 \\
\hline $26-09-16$ & 3717 & 2491 \\
\hline $27-09-16$ & 1822 & 4344 \\
\hline $28-09-16$ & 3870 & 2073 \\
\hline
\end{tabular}




\begin{tabular}{|c|c|c|}
\hline 29-09-16 & 3643 & 1775 \\
\hline $30-09-16$ & 4017 & 2028 \\
\hline 01-10-16 & 3178 & 1814 \\
\hline $02-10-16$ & 3309 & 1672 \\
\hline $03-10-16$ & 3084 & 1978 \\
\hline $04-10-16$ & 2398 & 2008 \\
\hline $05-10-16$ & 3018 & 2326 \\
\hline $06-10-16$ & 2742 & 1468 \\
\hline $07-10-16$ & 3289 & 1841 \\
\hline $08-10-16$ & 4039 & 3078 \\
\hline $09-10-16$ & 3790 & 2299 \\
\hline $10-10-16$ & 4205 & 3666 \\
\hline $11-10-16$ & 3903 & 2574 \\
\hline $12-10-16$ & 3998 & 3379 \\
\hline $13-10-16$ & 4279 & 3247 \\
\hline $14-10-16$ & 4198 & 2705 \\
\hline $15-10-16$ & 3858 & 2442 \\
\hline $16-10-16$ & 3868 & 2120 \\
\hline $17-10-16$ & 1502 & 954 \\
\hline 18-10-16 & 3769 & 2150 \\
\hline 19-10-16 & 4255 & 2705 \\
\hline $20-10-16$ & 3897 & 2938 \\
\hline $21-10-16$ & 3441 & 2195 \\
\hline $22-10-16$ & 3144 & 2090 \\
\hline $23-10-16$ & 3035 & 2312 \\
\hline 24-10-16 & 2775 & 2688 \\
\hline $25-10-16$ & 3575 & 2966 \\
\hline $26-10-16$ & 3485 & 2145 \\
\hline $27-10-16$ & 3813 & 2492 \\
\hline $28-10-16$ & 2918 & 1760 \\
\hline $29-10-16$ & 3530 & 2753 \\
\hline $30-10-16$ & 3596 & 2520 \\
\hline $31-10-16$ & 3365 & 2528 \\
\hline Total & 194,753 & 132,374 \\
\hline
\end{tabular}

Since in the actual elections, a person can vote only once. We have also applied a similar restriction, that only one tweet would be considered per person. The reason for this restriction was that nowadays many companies and agencies are being hired by the candidates in order to make the analysis bias. To rule out this anomaly, we had simply used the coding skills that if a person who tweeted multiple times, then the first tweet by that person would be considered for evaluation of results. Table 3 shows an example how this restriction works. In this "Roy" has tweeted 3 tweets, while "Sheral" has tweeted 2 tweets. So we set flag '1' for all tweets except the initial/first tweet. So for "Roy" and "Sheral" only one tweet will be counted, hence eliminating the effect of multiple tweets. 
Table 3. Example for applying restriction of one tweet per person

\begin{tabular}{|l|l|l|c|}
\hline Sr. No. & \multicolumn{1}{|c|}{ Tweet } & Sender & Flag \\
\hline 1 & I support Donald Trump & Roy & 0 \\
\hline 2 & Trump you are my hero & Roy & 1 \\
\hline 3 & Hillary we win this elections & Sheral & 0 \\
\hline 4 & Trump: Make US Great again & Roy & 1 \\
\hline 5 & Hillary we support you & Sheral & 1 \\
\hline
\end{tabular}

After applying this restriction, we were left with 136,192 (41.64\%) tweets, while 190,935 (58.36\%) duplicate tweets were removed. This highlights an important point that the number of people posting multiple tweets was quite high. Out of the 136,192 tweets collected from the USA, 81,946 (60.16\%) of tweet mentions were in favor of Mr. Donald Trump, while 54,246 (39.84\%). Table 4 shows the daily tweet collection for both candidates after applying the restriction of one tweet per person. Our entire experimentation was to be dependent on these 136,192 tweets.

Table 4. Daily tweet collection (With Restriction)

\begin{tabular}{|c|c|c|}
\hline Date & Donald Trump & Hillary Clinton \\
\hline $01-09-16$ & 1372 & 554 \\
\hline $02-09-16$ & 1097 & 564 \\
\hline $03-09-16$ & 1054 & 523 \\
\hline $04-09-16$ & 892 & 451 \\
\hline $05-09-16$ & 966 & 506 \\
\hline $06-09-16$ & 1540 & 1529 \\
\hline $07-09-16$ & 1013 & 600 \\
\hline $08-09-16$ & 1302 & 823 \\
\hline 09-09-16 & 1063 & 594 \\
\hline $10-09-16$ & 832 & 668 \\
\hline $11-09-16$ & 926 & 1686 \\
\hline $12-09-16$ & 1040 & 718 \\
\hline $13-09-16$ & 927 & 682 \\
\hline 14-09-16 & 1073 & 712 \\
\hline $15-09-16$ & 1306 & 638 \\
\hline $16-09-16$ & 1547 & 1963 \\
\hline $17-09-16$ & 1257 & 607 \\
\hline $18-09-16$ & 1161 & 590 \\
\hline 19-09-16 & 945 & 641 \\
\hline $20-09-16$ & 1163 & 690 \\
\hline $21-09-16$ & 1277 & 2058 \\
\hline $22-09-16$ & 1237 & 643 \\
\hline $23-09-16$ & 1219 & 583 \\
\hline $24-09-16$ & 1119 & 542 \\
\hline $25-09-16$ & 1171 & 627 \\
\hline $26-09-16$ & 1666 & 918 \\
\hline $27-09-16$ & 1016 & 1973 \\
\hline
\end{tabular}




\begin{tabular}{|c|c|c|}
\hline 28-09-16 & 1547 & 895 \\
\hline 29-09-16 & 1466 & 716 \\
\hline $30-09-16$ & 1623 & 756 \\
\hline 01-10-16 & 1315 & 725 \\
\hline $02-10-16$ & 1334 & 635 \\
\hline 03-10-16 & 1313 & 874 \\
\hline $04-10-16$ & 1038 & 772 \\
\hline $05-10-16$ & 1418 & 939 \\
\hline $06-10-16$ & 1299 & 643 \\
\hline $07-10-16$ & 1540 & 762 \\
\hline $08-10-16$ & 1786 & 1064 \\
\hline $09-10-16$ & 1679 & 918 \\
\hline $10-10-16$ & 1854 & 1506 \\
\hline $11-10-16$ & 1685 & 1009 \\
\hline $12-10-16$ & 1677 & 1100 \\
\hline $13-10-16$ & 1802 & 1111 \\
\hline $14-10-16$ & 1828 & 995 \\
\hline $15-10-16$ & 1655 & 963 \\
\hline $16-10-16$ & 1604 & 863 \\
\hline $17-10-16$ & 791 & 515 \\
\hline $18-10-16$ & 1500 & 900 \\
\hline $19-10-16$ & 1676 & 1120 \\
\hline $20-10-16$ & 1659 & 1193 \\
\hline $21-10-16$ & 1362 & 904 \\
\hline $22-10-16$ & 1285 & 793 \\
\hline $23-10-16$ & 1294 & 757 \\
\hline $24-10-16$ & 1213 & 892 \\
\hline $25-10-16$ & 1444 & 930 \\
\hline $26-10-16$ & 1593 & 944 \\
\hline $27-10-16$ & 1585 & 966 \\
\hline $28-10-16$ & 1360 & 888 \\
\hline $29-10-16$ & 1557 & 1068 \\
\hline $30-10-16$ & 1498 & 992 \\
\hline $31-10-16$ & 1485 & 1055 \\
\hline Total & 81,946 & 54,246 \\
\hline
\end{tabular}




\section{Results and Findings}

As mentioned earlier, volume of tweets is not the deciding factor for the victory of any specific candidate, we computed polarity (positive or negative) for each tweet by applying sentiment analysis. Sentiment analysis is the study of analyzing people's opinions, sentiments, evaluations, appraisals, attitudes and emotions towards entities such as products, services, organizations, individuals, issues, events, topics, and their attributes [2].

For this, we developed a classification model in WEKA 3.8 [17], which is open source software and consists of a collection of machine learning algorithms for data mining tasks. Further we applied support vector machines (SVM) which is a supervised machine learning approach for performing the sentiment analysis. The SVM is a learning machine for two-group classification problems that transforms the attribute space into multidimensional feature space using a kernel function to separate dataset instances by an optimal hyperplane [18]. The reason for building the model using SVM was that it is often regarded as one of the best classification algorithm [19].

The training data set was same as used by Kotzias et al. [20], which contains reviews and scores from three different datasets i.e. Amazon [21], IMDb [22], Yelp [23]. Each dataset contains a total of 500 positive and 500 negative sentences, so in total the dataset had 1500 positive and 1500 negative sentences. The data set contained two columns first the sentence and second the sentiment of each sentence in form of " 0 " (negative) and "1" (positive).

For classification, we used filtered classifiers, which enable us to build a classifier with a filter of our choice. As discussed earlier SVM is used as classifier while "StringToWordVector" is used as a filter which convert a string attribute to a vector that represents word occurrence frequencies. In addition to this we used 10 fold cross validation which is also known as rotation estimation to analyze how a predictive model would perform on an unknown dataset. The training set got an efficiency of $79.26 \%$, this means 2378 instances from the training set were correctly classified while 622 instances were incorrectly classified. According to the confusion matrix 1191 negative instances (Class a) were correctly classified while 1167 positive instances (Class b) were correctly classified. The detailed results along with confusion matrix are shown in Figure 2, while Figure 3 shows the graph showing area under the curve $(\mathrm{ROC}=0.793)$. 


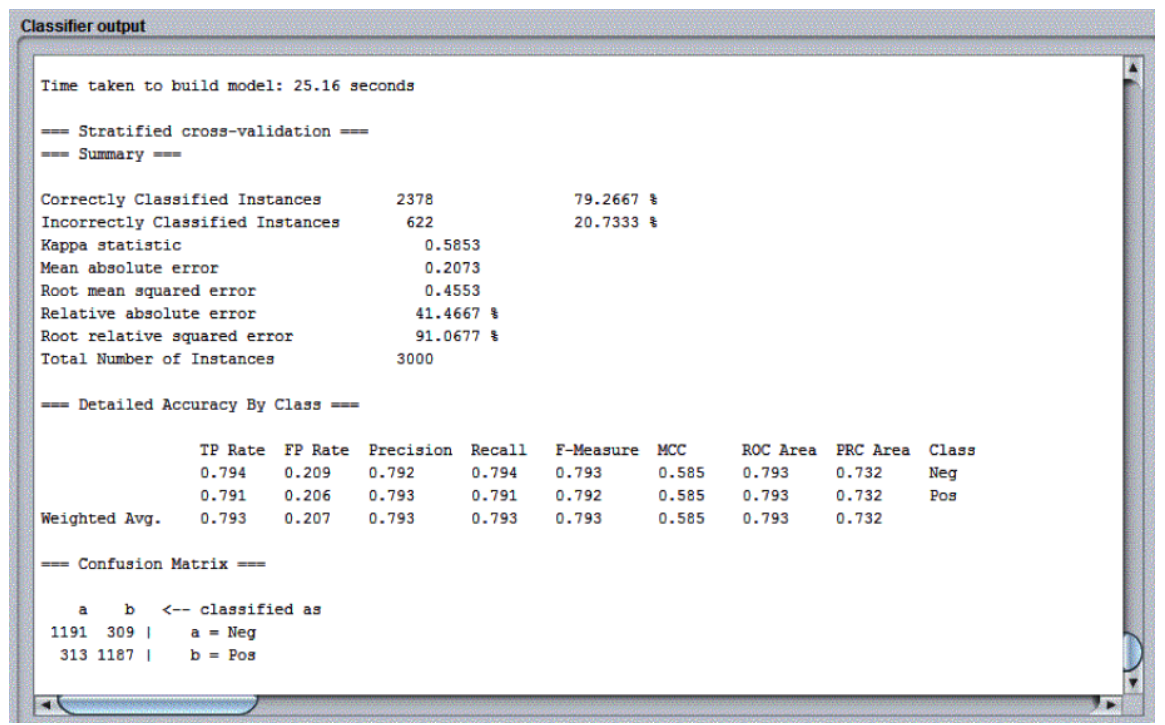

Fig. 2. Results of Classification Model

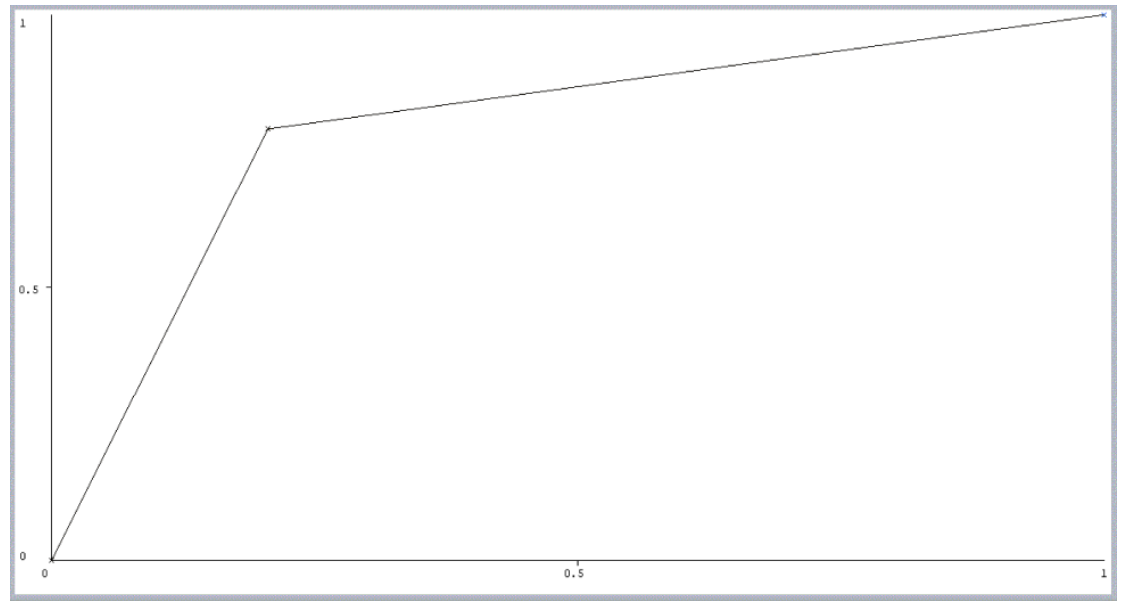

Fig. 3. Area under the Curve $(\mathrm{ROC}=0.793)$

For testing set, we used the tweets collected from Twitter. Before testing we preprocessed the data in order to remove unwanted Html tags, web links and special symbols (, " ! '; : @ \#) so that we should not get biased results. The task of data preprocessing was performed in an automated fashion. Once preprocessing was done, we passed the testing set through the classification model developed earlier and it gave us the classification results i.e. polarity of each tweet. From these results we calculated net positive score (NPS), which is simply the difference between the total number of positive tweets and total number of negative tweets received by a candidate. The results of the same have been shown in Table 5 . 
Table 5. Result of Sentiment Analysis for both candidates

\begin{tabular}{|l|l|l|}
\hline \multirow{2}{*}{} & \multicolumn{2}{|c|}{ Number of Tweets } \\
\cline { 2 - 3 } & Donald Trump & Hillary Clinton \\
\hline Positive & 42518 & 27582 \\
\hline Negative & 39428 & 26664 \\
\hline Net Positive Score (NPS) & 3090 & 918 \\
\hline
\end{tabular}

Out of the total 81,946 tweet for Donald Trump got, 42,518 (51.88\%) tweets were positive and $27,582(48.12 \%)$ tweets were negative. Similarly out of the total 54,246 tweets Hillary Clinton got, 27,582 (50.84\%) tweets were positive and 26,664 (49.16\%) tweets were negative. The net positive score (NPS) of Donald Trump was observed to be significantly higher than that of Hillary Clinton. Based upon our experimental results it was evident that Donald Trump would be winning the 2016 US Presidential Elections. Figure 4 shows the results of the same in graphical form.

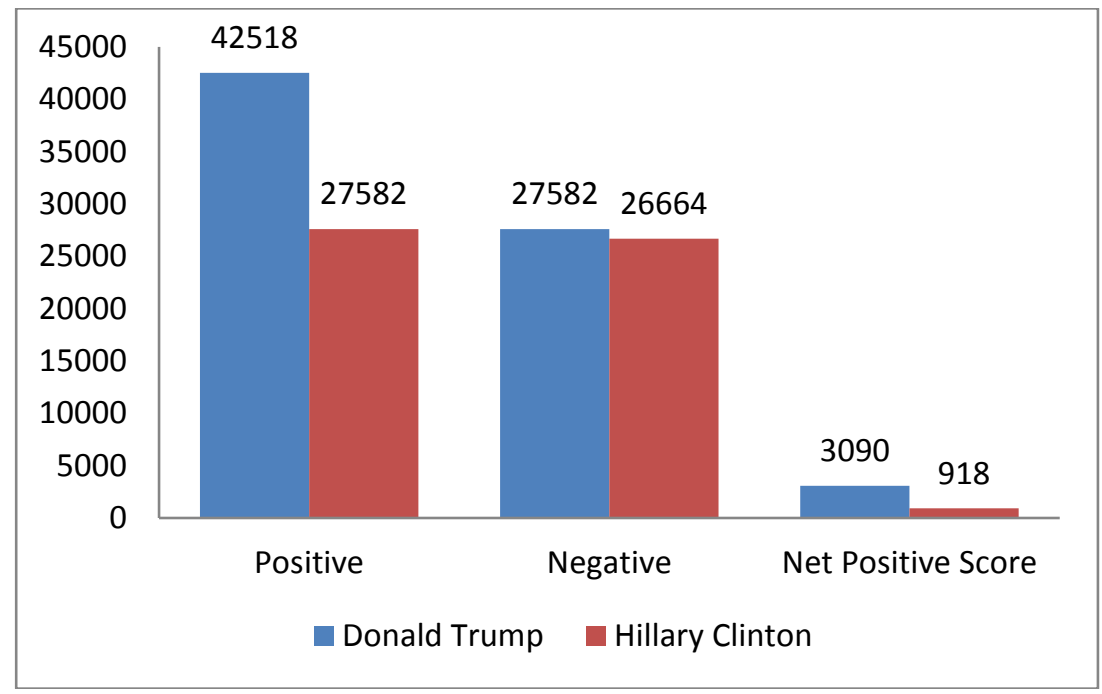

Fig. 4. Final Results

\section{Conclusions and Future Work}

Predicting an event is always an uphill task. There are lots of factors that ought to be considered for making a truthful prediction. The aim of this paper was to predict the winner of 2016 US Presidential Elections. For this we collected data from Twitter. Further we applied a restriction that only one tweet per person will be considered for evaluation. Finally, we build a classification model in WEKA using SVM for performing sentiment analysis. Based upon the results of sentiment analysis we calculated the NPS. The results of our experiments clearly indicate that Donald Trump would be winning the 2016 US Presidential Elections. 
Our experiments gave us the probability that the winner will be Donald Trump, however the actual winner in the US presidential election is based on the electoral vote and not the percentage of votes, and we should build a mathematical model that can convert the results of sentiment analysis into electoral votes which indeed will be our future aim.

\section{References}

1. Statisticbrain Twitter Facts, "http://www.statisticbrain.com/Twitter-statistics/"

2. Liu, Bing. "Sentiment analysis and opinion mining."Synthesis lectures on human language technologies 5, no. 1 (2012): 1-167. doi: 10.2200/S00416ED1V01Y201204HLT016

3. IMF Report, "http://www.imf.org/external/pubs/ft/weo/2015/01/weodata/groups.htm"

4. CIA Internet User Report,"https://www.cia.gov/library/publications/resources/theworld-factbook/rankorder/2153rank.html"

5. Statisticbrain US Twitter Facts, "https://www.statista.com/statistics/274564/monthlyactive-Twitter-users-in-the-united-states/"

6. Tumasjan, Andranik, Timm Oliver Sprenger, Philipp G. Sandner, and Isabell M. Welpe (2010). "Predicting elections with Twitter: What 140 characters reveal about political sentiment." ICWSM 10: 178-185.

7. Jungherr, Andreas (2013). "Tweets and votes, a special relationship: The 2009 federal election in germany." In Proceedings of the 2nd workshop on Politics, elections and data, pp. 5-14. Doi: 10.1145/2508436.2508437

8. Gayo Avello, Daniel, Panagiotis T. Metaxas, and Eni Mustafaraj. "Limits of electoral predictions using twitter." In Proceedings of the Fifth International AAAI Conference on Weblogs and Social Media. Association for the Advancement of Artificial Intelligence, 2011.

9. Gayo-Avello, Daniel. "I Wanted to Predict Elections with Twitter and all I got was this Lousy Paper A Balanced Survey on Election Prediction using Twitter Data." arXiv preprint arXiv:1204.6441 (2012).

10. DiGrazia, Joseph, Karissa McKelvey, Johan Bollen, and Fabio Rojas. "More tweets, more votes: Social media as a quantitative indicator of political behavior." PloS one 8, no. 11 (2013): e79449. doi: 10.1371/journal.pone.0079449

11. Franch, Fabio. "(Wisdom of the Crowds) 2: 2010 UK election prediction with social media." Journal of Information Technology \& Politics 10, no. 1 (2013): 57-71. doi: 10.1080/19331681.2012.705080

12. Ceron, Andrea, Luigi Curini, Stefano M. Iacus, and Giuseppe Porro. "Every tweet counts? How sentiment analysis of social media can improve our knowledge of citizens' political preferences with an application to Italy and France." New Media \& Society 16, no. 2 (2014): 340-358. doi: 10.1177/1461444813480466

13. Caldarelli, Guido, Alessandro Chessa, Fabio Pammolli, Gabriele Pompa, Michelangelo Puliga, Massimo Riccaboni, and Gianni Riotta. "A multi-level geographical study of Italian political elections from Twitter data." PloS one 9, no. 5 (2014): e95809. doi: 10.1371/journal.pone.0095809

14. Burnap, Pete, Rachel Gibson, Luke Sloan, Rosalynd Southern, and Matthew Williams. "140 characters to victory?: Using Twitter to predict the UK 2015 General Election." Electoral Studies 41 (2016): 230-233. doi: 10.1016/j.electstud.2015.11.017 
15. Visual Studio 2012, "https://www.visualstudio.com/en-us/downloads/download-visualstudio-vs.aspx"

16. Tweetinvi API, "https://www.nuget.org/packages/TweetinviAPI/"

17. Eibe Frank, Mark A. Hall, and Ian H. Witten (2016). The WEKA Workbench. Online Appendix for "Data Mining: Practical Machine Learning Tools and Techniques", Morgan Kaufmann, Fourth Edition, 2016.

18. Hearst, Marti A., Susan T. Dumais, Edgar Osuna, John Platt, and Bernhard Scholkopf. "Support vector machines." IEEE Intelligent Systems and their Applications 13, no. 4 (1998): 18-28. doi: 10.1109/5254.708428

19. Petrova, Natalia V., and Cathy H. Wu. "Prediction of catalytic residues using Support Vector Machine with selected protein sequence and structural properties." BMC bioinformatics 7, no. 1 (2006): 312. doi: 10.1186/1471-2105-7-312

20. Kotzias, Dimitrios, Misha Denil, Nando De Freitas, and Padhraic Smyth. "From group to individual labels using deep features." In Proceedings of the 21th ACM SIGKDD International Conference on Knowledge Discovery and Data Mining, pp. 597-606. ACM, 2015. doi: 10.1145/2783258.2783380

21. McAuley, Julian, and Jure Leskovec. "Hidden factors and hidden topics: understanding rating dimensions with review text." In Proceedings of the 7th ACM conference on Recommender systems, pp. 165-172. ACM, 2013. doi: 10.1145/2507157.2507163

22. Maas, Andrew L., Raymond E. Daly, Peter T. Pham, Dan Huang, Andrew Y. Ng, and Christopher Potts. "Learning word vectors for sentiment analysis." In Proceedings of the 49th Annual Meeting of the Association for Computational Linguistics: Human Language Technologies-Volume 1, pp. 142-150. Association for Computational Linguistics, 2011.

23. Yelp Dataset, "https://www.yelp.com/dataset_challenge" 